\title{
Serial Tissue Expansion at the Same Site in Pediatric Patients: Is the Subsequent Expansion Faster?
}

\author{
Moon Ki Lee ${ }^{1}$, Seong Oh Park ${ }^{1}$, Tae Hyun Choi ${ }^{1,2}$ \\ ${ }^{1}$ Department of Plastic and Reconstructive Surgery, Institute of Human-Environment Interface Biology, Seoul National University College of \\ Medicine, Seoul; ${ }^{2}$ Department of Pediatric Plastic and Reconstructive Surgery, Seoul National University Children's Hospital, Seoul, Korea
}

Background Serial tissue expansion is performed to remove giant congenital melanocytic nevi. However, there have been no studies comparing the expansion rate between the subsequent and preceding expansions. In this study, we analyzed the rate of expansion in accordance with the number of surgeries, expander location, expander size, and sex.

Methods A retrospective analysis was performed in pediatric patients who underwent tissue expansion for giant congenital melanocytic nevi. We tested four factors that may influence the expansion rate: The number of surgeries, expander location, expander size, and sex. The rate of expansion was calculated by dividing the 'inflation amount' by the 'expander size'.

Results The expansion rate, compared with the first-time group, was 1.25 times higher in the second-or-more group $(P=0.04)$ and 1.84 times higher in the third-or-more group $(P<0.01)$. The expansion rate was higher at the trunk than at other sites $(P<0.01)$. There was a tendency of lower expansion rate for larger expanders $(P=0.03)$. Sex did not affect the expansion rate.

Conclusions There was a positive correlation between the number of surgeries and the expansion rate, a positive correlation between the expander location and the expansion rate, and a negative correlation between the expander size and the expansion rate.

Keywords Nevus, pigmented / Tissue expansion / Tissue expansion devices / Skin neoplasms
Correspondence: Tae Hyun Choi Department of Plastic and Reconstructive Surgery, Seoul National University College of Medicine, 101 Daehak-ro, Jongno-gu, Seoul 03080, Korea

Tel: +82-2-2072-1978

Fax: +82-2-766-5829

E-mail: psthchoi@snu.ac.kr

This research was supported by a grant through the Disaster and Safety Management Institute (MPSSCG-2016-02), Center for Research and Development of Police science and Technology and Korean National Police Agency (PA-H000001), and the Seoul National University Hospital Research Fund (26-2015-0030), Republic of Korea.

This article was presented at The 74th Congress of the Korean Society of Plastic and Reconstructive Surgeons on November 17-20, 2016 in Seoul, Korea.

\section{INTRODUCTION}

In adults, the size of congenital melanocytic nevus is classified using the length of its diameter. Nevus with a diameter of up to $1.5 \mathrm{~cm}$ is considered as small, between 1.5 and $19.9 \mathrm{~cm}$ as medi- um, greater than $20 \mathrm{~cm}$ as large, and greater than $50 \mathrm{~cm}$ as giant [1]. In pediatrics, on the other hand, the size is classified depending on the location. Nevus on the head and neck area with a size equivalent to the palm of the patient is considered as giant. On the body, nevus with a size greater than $30 \%$ of the surface 
of the body or greater than $20 \mathrm{~cm}$ in diameter is considered as giant [2].

Giant congenital melanocytic nevus is treated not only for its malignant potential, but also for its associated psychological distress. There are two treatment options: Nonexcisional (i.e., dermabrasion, laser ablation, curettage, chemical peel) and excisional (i.e., staged or en bloc excision with primary closure, skin graft, flap, or tissue expansion). Tissue expansion surgery-an excisional approach - has been regarded as the first-line treatment for giant congenital melanocytic nevi due primarily to the similar qualities between the created tissue and the normal skin. Tissue expansion can offer improved color matching, better texture, and minimum malformation of the donor site [3].

Tissue expansion is generally performed over a 2 - to 6-month period, with inflation performed on a weekly basis. The maximum amount of the lesion is excised, and the expanded skin is maneuvered to fill the defect. If there are any remnants of the nevus, tissue expanders should be reinserted for a subsequent expansion surgery [4].

There are some doubts on whether subsequent expansions become more difficult, which means slower, or easier, which means faster than the preceding expansion. To the best of our knowledge, there have not been any studies in the literature that investigated the level of difficulty for subsequent expansions. Herein, we focused on the expansion rate to determine how well the skin has expanded in accordance with the number of surgeries, expander location, expander size, and sex.

\section{Fig. 1. First set of surgery}

(A) A preoperative photograph. At the time of tissue expander insertion, the patient was 14-month-old. The inserted expander was $280 \mathrm{~mL}$ and rectangular shape. Inflation was performed weekly from postoperative 19 days to postoperative 89 days, and the total amount was $505 \mathrm{~mL}$. (B) An immediate postoperative photograph. Tissue expander removal and local flap were performed when the patient was 18-month-old. Lower part of the nevus was excised.
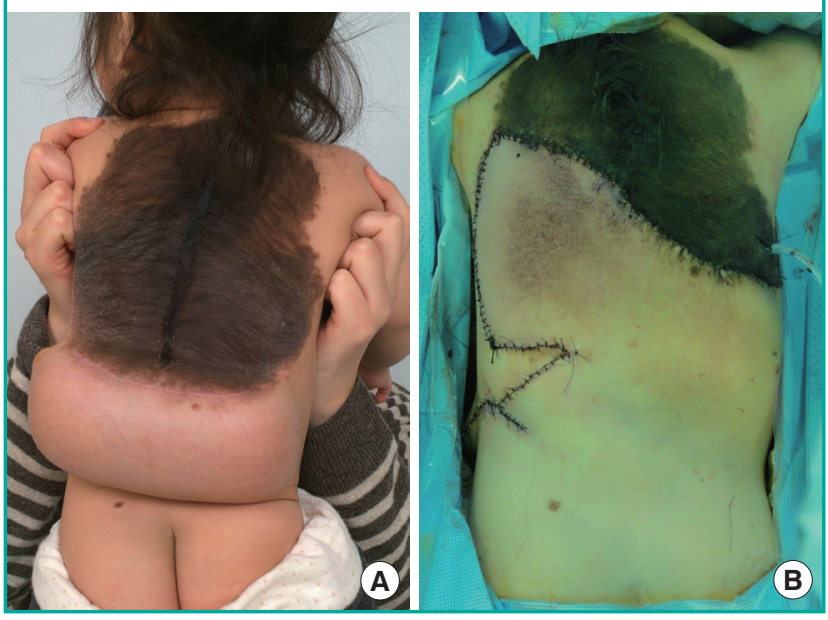

\section{METHODS}

\section{Patients}

A retrospective analysis was performed in 77 pediatric patients who underwent tissue expansion for giant congenital melanocytic nevi between May 2009 and July 2015. All expanders were placed under the galea in the scalp, subcutaneous fat in the face, and suprafascial space in the other regions. The expander size was selected based on the areas of the nevus and adjacent normal skin. Subsequent tissue expansion should be performed on the same flap (previously expanded) as the preceding surgery (Figs. 1-3). Operations that were performed on a different site were considered as a first surgery.

The postoperative inflation protocol was the same for all patients. Patients who underwent tissue expander insertion began inflation at 3 weeks postoperatively. On a weekly basis, normal saline was used to inflate the skin as much as possible without skin pallor. Inflation was performed until reaching the desired volume. Our patient cohort included 59 patients who underwent this full course. The study was approved by the local Institutional Review Board (IRB no. 1012-060-344).

\section{Definition of expansion rate}

Inflation of the tissue expander was performed on a weekly basis. In most cases, the desired volume was 1.5-2 times bigger than the inserted expander. For each inflation, injection of normal saline was stopped when the skin turned pale. The amount

Fig. 2. Second set of surgery

(A) A preoperative photograph. At the time of tissue expander insertion, the patient was 27-month-old. The inserted expander was 380 $\mathrm{mL}$ and rectangular shape. Inflation was performed weekly from postoperative 20 days to postoperative 90 days, and the total amount was $869 \mathrm{~mL}$. (B) An immediate postoperative photograph. Tissue expander removal and local flap were performed when the patient was 31-month-old. Expanded site was the same as that of the first surgery and lower medial part of the remnant nevus was excised.
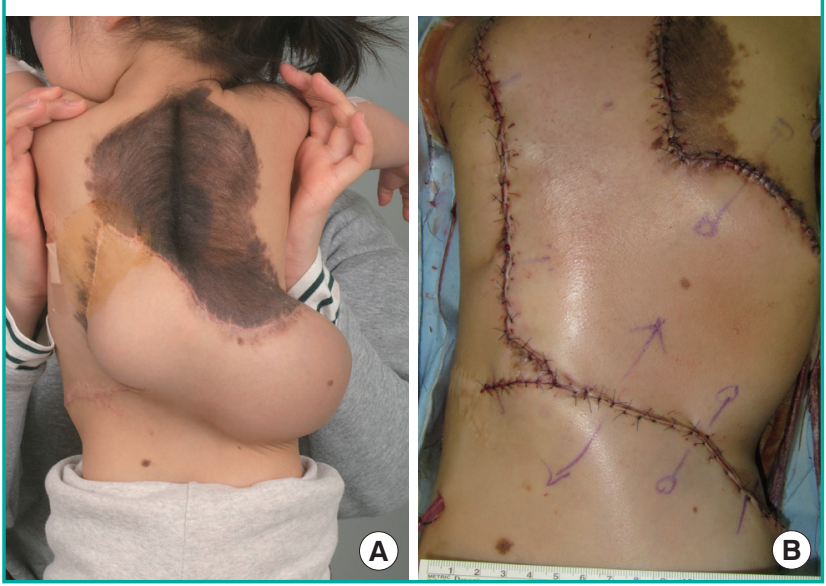
of single saline injection varied from patient to patient. This amount was larger if the skin showed a tendency to expand more easily. It was also larger if the expander itself was bigger. Therefore, we calculated the "expansion rate (\%)" by dividing the inflation amount at one time $(\mathrm{mL})$ by the expander size $(\mathrm{mL})$. A higher expansion rate suggests a larger amount of normal saline compared with the expander volume, which could have been injected at one time without skin pallor. If this tendency was to be maintained for the entire period of the expansion, it would be possible to obtain the desired volume at a faster rate.

\section{Affecting factors and statistics}

We identified four possible factors that may affect the expansion rate: The number of surgeries, which ranged from one to six; the expander insertion site, which consisted of the scalp, face, supraclavicle, trunk, upper limb, and lower limb; the expander size; and sex [5].

The distribution of expansion rate was right-skewed and expansion rate was log-transformed. A mixed-model was used because one patient underwent multiple surgeries at several different sites. To be more specific, patients were considered as a random effect and variables were considered as a fixed effect. Relative ratio was used for the comparison between factors in logtransformed data. A value of $\mathrm{P}<0.05$ was considered to indicate statistical significance and all statistical analyses were performed using SAS V.9.2 (SAS Institute, Cary, NC, USA).

\section{Fig. 3. Third set of surgery}

(A) A preoperative photograph. At the time of tissue expander insertion, the patient was 4-year-old. The inserted expander was 180 $\mathrm{mL}$ and rectangular shape. Inflation was performed weekly from postoperative 18 days to postoperative 109 days, and the total amount was $659 \mathrm{~mL}$. (B) An immediate postoperative photograph. Tissue expander removal and local flap were performed 4 months later. Expanded site was the same as that of the previous surgeries, and the remnant nevus was totally excised.
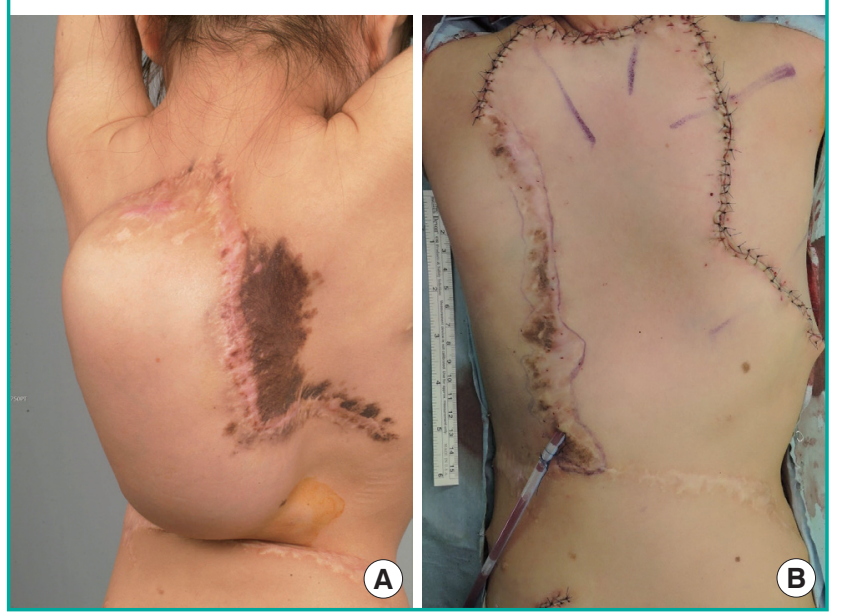

\section{RESULTS}

\section{Demographic results}

The demographic characteristics are presented in Tables 1 and 2. Among 77 pediatric patients, we excluded 3 patients who did not undergo a typical inflation course due to postoperative complications. Additional 2 patients were excluded for having unclear medical records. Moreover, patients who received subsequent expander insertions by different surgeons ( 8 patients) and those who received surgery with a non-rectangular-shaped expander ( 5 patients) were also excluded. Fifty-nine patients aged between one and 15 underwent 83 tissue expansion surgeries in total; 58 of these surgeries were first expansion, and 25 were subsequent (second or more) expansions. Although one patient underwent the first and second surgery at another hospital, the requirement was fulfilled from the third surgery, which was performed at our hospital. Among the 83 cases, 10 cases were performed on the scalp, 18 on the face, 2 on the suprascapular area, 40 on the trunk, 6 on the upper limb, and 7 on the lower limb (Table 1). The expander size ranged from 8 to 700 $\mathrm{mL}$ (Table 2). 38 cases were performed in male patients, and 45 in female patients.

\section{Affecting factors to expansion rate}

Increasing the number of surgeries increased the expansion rate with statistical significance $(\mathrm{P}<0.01)$. The expansion rate in the first-surgery group and the second-or-more group was $19 \%$ and

\section{Table 1. Number of operations and site of expander insertion}

\begin{tabular}{|lclc|}
\hline No. of operations & Cases & Insertion site & Cases \\
\hline 1 & 58 & Scalp & 10 \\
2 & 17 & Face & 18 \\
3 & 4 & Supraclavicular & 2 \\
4 & 2 & Trunk & 40 \\
5 & 1 & Upper limb & 6 \\
6 & 1 & Lower limb & 7 \\
Total & 83 & Total & 83 \\
\hline
\end{tabular}

Table 2. Size of the expander

\begin{tabular}{|lc|}
\hline Expander size $(\mathrm{mL})$ & Cases \\
\hline $8-16$ & 2 \\
$45-60$ & 12 \\
100 & 14 \\
$160-200$ & 17 \\
280 & 17 \\
$360-400$ & 12 \\
560 & 7 \\
700 & 2 \\
Total & 83 \\
\hline
\end{tabular}


$23 \%$, respectively. The expansion rate of the second-or-more group was 1.25 times larger than that of the first-surgery group with statistical significance $(\mathrm{P}=0.04)$ (Table 3$)$. In the third-ormore group, the rate increased by 1.84 -fold $(\mathrm{P}<0.01)$ (Table 4$)$. The expansion rate of the second-or-more group was higher than that of the first-surgery group at every week of inflation (Fig. 4).

Table 3. Relative ratio of the expansion rate for first and second-or-more expansions

\begin{tabular}{|c|c|c|c|}
\hline \multirow{2}{*}{ Characteristic } & \multicolumn{2}{|c|}{ No. of operations } & \multirow{2}{*}{ P-value $e^{a)}$} \\
\hline & $1 \mathrm{st}$ & $\geq 2$ nd & \\
\hline No. & 58 & 25 & - \\
\hline Expansion rate $(\%)^{b)}$ & $18.75(16.42,21.41)$ & $23.44(19.13,28.71)$ & - \\
\hline Relative ratio & 1 (Reference) & 1.25 & 0.04 \\
\hline
\end{tabular}

The expansion site significantly affected the expansion rate $(\mathrm{P}<0.01)$. Compared with the group that underwent expander insertion at the scalp, the expansion rate for groups that underwent expansion at the trunk, supraclavicular area, upper limb, and lower limb was 1.88-fold $(\mathrm{P}<0.01), 1.01$-fold $(\mathrm{P}=0.94)$, 1.29-fold $(\mathrm{P}=0.03)$, and 1.24-fold $(\mathrm{P}=0.34)$ higher, respectively (Fig. 5).

The data also showed that the expander size had a significant influence on the expansion rate $(\mathrm{P}=0.03)$. When a larger expander was inserted, the expansion rate tended to be lower. The expansion rate of the largest expander group $(700 \mathrm{~mL})$ was 0.26 times that of the smallest expander group $(8-16 \mathrm{~mL})$. Fig. 6 shows these results.

There was no significant difference between the sexes $(\mathrm{P}=$ 0.45 ) with respect to the expansion rate. The expansion rate of males was 23\% (with a lower estimate of 17.58 and an upper estimate of 28.22) and of females was $21 \%$ (with a lower estimate of 15.74 and an upper estimate of 26.70 ).

Table 4. The relative ratios of the expansion rate for first, second, and third-or-more expansions

\begin{tabular}{|c|c|c|c|c|}
\hline \multirow{2}{*}{ Characteristic } & \multicolumn{3}{|c|}{ No. of operations } & \multirow{2}{*}{ P-value ${ }^{a)}$} \\
\hline & $1 \mathrm{st}$ & 2nd & $\geq 3$ rd & \\
\hline No. & 58 & 17 & 8 & - \\
\hline Expansion rate $(\%)^{b)}$ & $18.75(16.42,21.41)$ & $19.98(15.99,24.96)$ & $34.28(24.88,47.24)$ & - \\
\hline Relative ratio & 1 (Reference) & 1.07 & 1.84 & $<0.01$ \\
\hline
\end{tabular}

\section{Fig. 4. Tissue expansion rate of the two groups}

Horizontal axis is the week of inflation. Inflation is performed weekly and except for the second week of inflation, the patients who had underwent more than two surgeries showed a higher average expansion rate than those who had underwent only the first surgery. This graph shows that the tendency for a higher average expansion rate of subsequent expansion group continues during the whole inflation period. 1st, first expansion group, blue line; $\geq 2$ nd, second-or-more expansion group, red line; Weeks, the week inflation was done (week 1 means the first week of inflation).

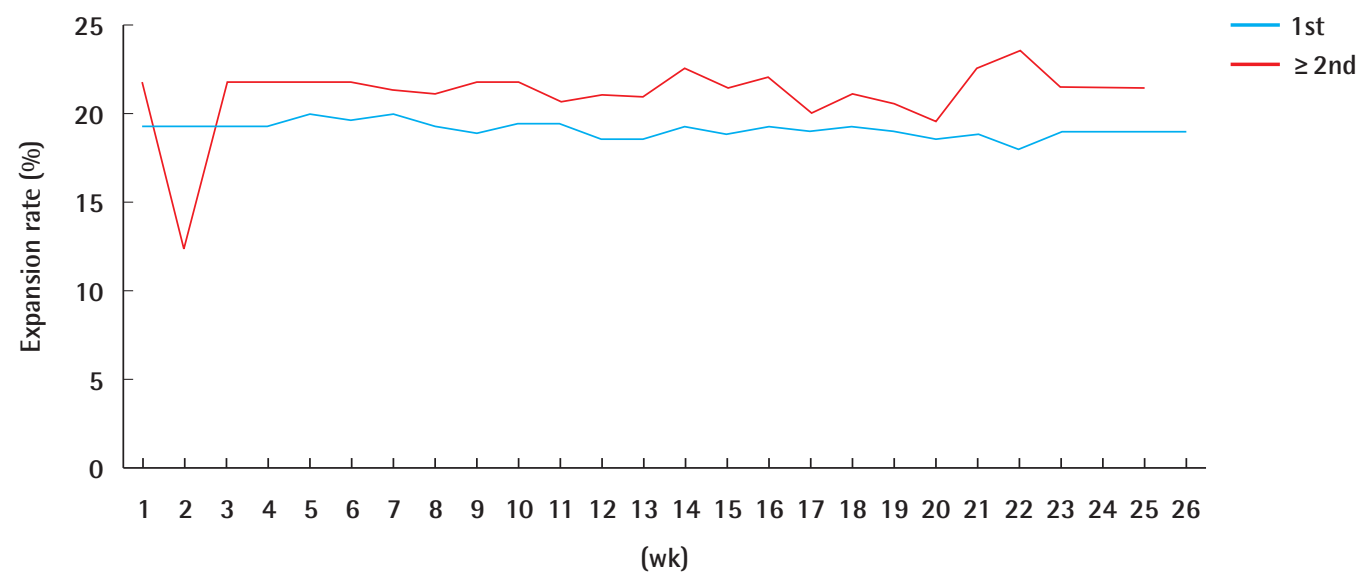




\section{Fig. 5. Relationship between tissue expander insertion sites and expansion rate}

Compared to the group underwent expander insertion at the scalp, the group that underwent expander insertion at the trunk showed a 1.88-fold higher expansion rate $(P<0.01)$. The supraclavicular area showed a 1.01-fold higher expansion rate $(P=0.94)$, and the upper limb showed a 1.29fold $(P=0.30)$ and the lower limb showed a 1.24-fold $(P=0.34)$ higher expansion rate. Expansion rate was estimated by the univariable mixed linear model. Bars indicate the standard error. *Statistical significance $(P<0.05)$.

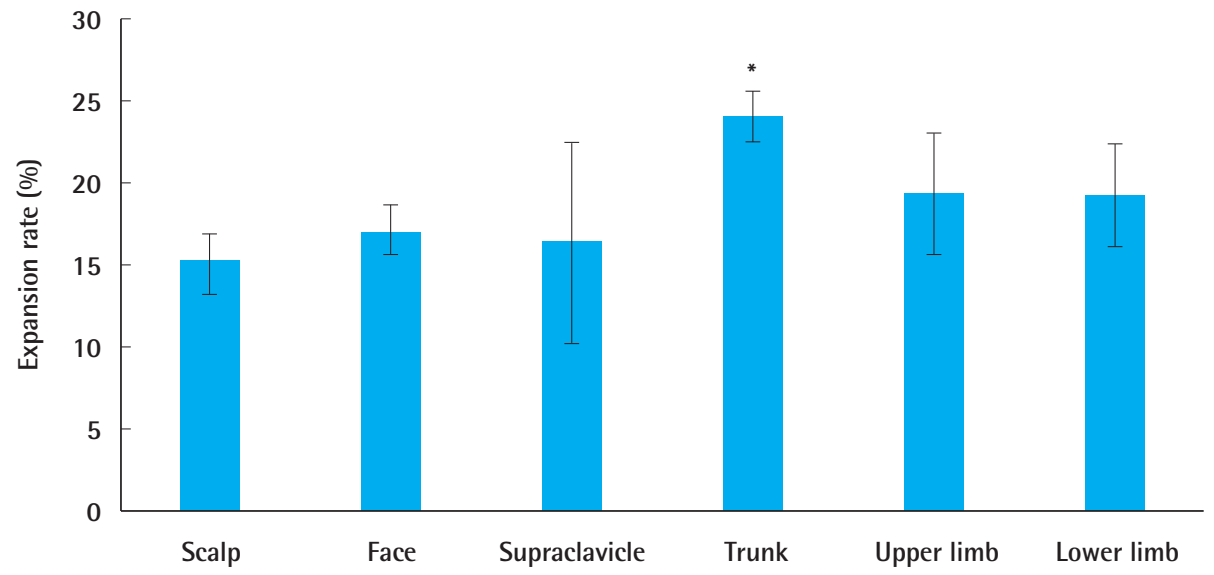

\section{Fig. 6. Relationship between expander size and expansion rate}

Larger expander shows lower expansion rate. Compared with the smallest expander group (8-16 $\mathrm{mL})$, the largest expander group (700 $\mathrm{mL}$ ) showed a 0.26 times expansion rate $(P<0.01)$. Expansion rate was estimated by the univariable mixed linear model. Bars indicate the standard error. *Statistical significance $(\mathrm{P}<0.05)$.

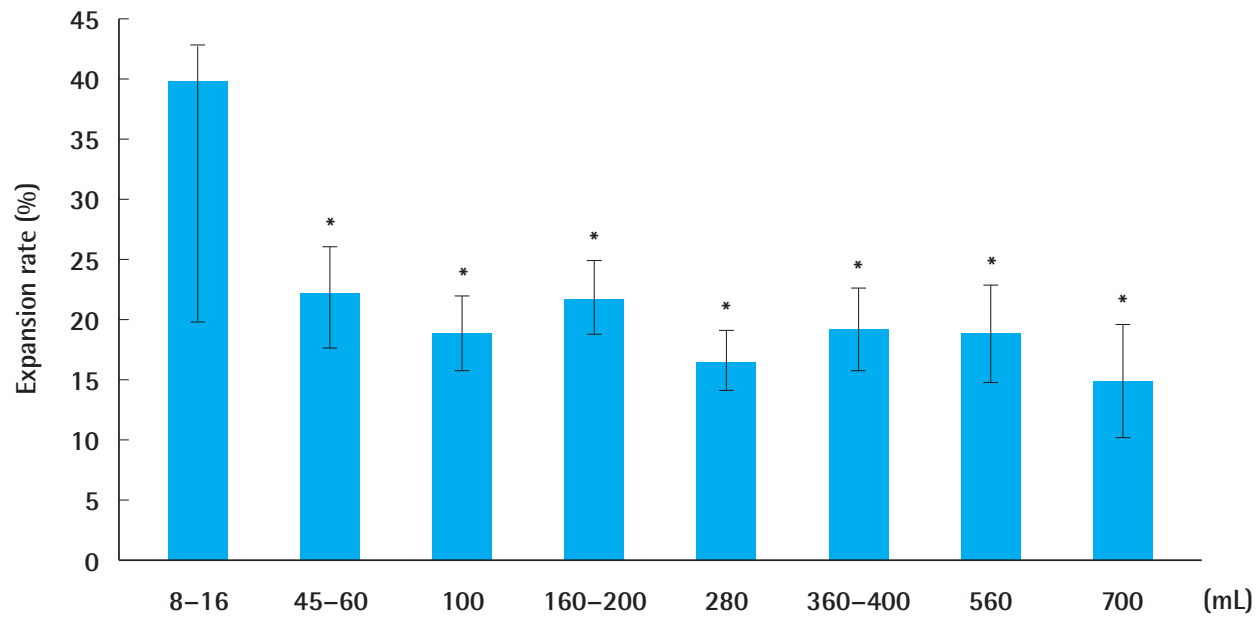

\section{DISCUSSION}

There have been two conflicting hypotheses regarding the speed of subsequent expansions compared with the preceding expansion. First, subsequent expansions are slower and more difficult than the preceding expansion. The logic behind this is that the dermis becomes thinner after the initial expansion, and a thin dermis cannot afford the strength of subsequent expansions. Second, the subsequent expansion is faster and easier than the preceding expansion. The collagen and elastic fibers of the dermis are broken down from the preceding expansion, making it possible for the skin to be more easily expanded in subsequent expansions. Considering this dichotomy, the purpose of this study was to test which of the two hypotheses is more plausible. We defined the expansion rate to test this hypothesis. Although there was no consideration for a time scale in the definition of the expansion rate, the calculation reflected the velocity of expansion. Easier and faster expansion meant reaching the desired volume at a faster pace.

We selected four possible factors that may affect the expansion 
rate. The expansion rate can either be proportional or inversely proportional to the number of surgeries and be influenced by the insertion site. The expander size was included in the definition of the expansion rate, but there may still be a possibility that it affects the expansion rate. It is possible that female patients show a higher expansion rate because they have softer skin. Therefore, sex was considered to be a factor influencing the expansion rate. Moreover, it has also been established that the shape of the expander can affect the expansion rate; hence, we selected patients who underwent expansion with rectangular expanders [5]. According to our data, the number of expansions showed a positive correlation with the expansion rate. In other words, an increased number of expansions was associated with a higher expansion rate. A higher expansion rate indicates greater flexibility. We assumed that when a site undergoes an expander insertion once, its flexibility increases as the elastic fiber gets broken down to some degree and collagen fiber aligns in a favorable position for expansion. Conventional tissue expansion may result in epidermal hypertrophy, decreased dermal thickness, muscle, and adipose thickness, as well as bone resorption. During the expansion procedure, the dermis decreases rapidly in thickness [6]. Dermal collagen deposition increases and collagen fibers realign along the tension line. Jeong et al. [7] performed a histological examination of before and after skin expansion, demonstrating an increase in the porosity of the dermal layer and an uniaxial arrangement of the collagen fibers. Fragmentation of the elastic fiber and flattening of the dermal papillae also occur in response to tissue expansion [8]. Based on this histological knowledge, we hypothesized that the dermal tissue becomes more flexible from expansion - by collagen realignment and elastic fiber fragmentation - and consequently, making it favorable for an additional expansion procedure. Tissue growth is known to contribute to the biomechanical change of expanded tissue. Using the model designed by Zollner et al. [9], we can calculate how much growth would occur. Our results showed that more tissue growth might occur if the expanded flap is a previously expanded site. It can be assumed that the critical threshold of the flap decreases as the number of expansions increases, and further computational model studies would be worthwhile to be performed to confirm this assumption.

Moreover, as indicated by our analysis, the insertion site was also an important factor influencing the expansion rate. The group that underwent expander insertion at the trunk showed the highest expansion rate. It has been well established that various anatomical regions have different mechanical properties [10]. The difference in the expansion rate between the insertion sites is likely due to the various fiber characteristics specific to each site. The elastic fibers of the trunk are more loosely aligned than that of the scalp and extremities. This explains why the trunk is associated with the highest expansion rate. According to the study regarding the biological elasticity using maximum skin deformation, skin characteristics differ substantially across various anatomical sites, as well as between sexes. Locations where the skin is directly superior to the boney surface were least elastic. Moreover, the anterior surface of the lower leg exhibited the lowest elasticity (maximal deformation range from $0.583-0.712$ $\mathrm{mm})$, while the abdomen $(1.373-1.663 \mathrm{~mm})$, and lower back $(1.322-1.562 \mathrm{~mm})$ showed the highest elasticity [11]. These results coincide with our results. Because the trunk has loosely aligned elastic fibers and its soft tissues are loosely connected to the bony surface, it is associated with greater flexibility and can be expanded more easily and rapidly.

Additionally, the size of the expander showed a negative correlation with the expansion rate. When we inflate a larger expander, small percentages of normal saline can make the skin pale, which require more time to achieve the desired final volume. It is natural that a smaller expander can be expanded faster, and our results correlate with this logic.

However, sex did not have any effect on the expansion rate. Nedelec et al. studied the influence of sex on skin elasticity and found that females showed a greater skin elasticity than males. However, in this study, there was no significant difference between the two sexes. We believe that sex was not influential because our patients were pediatric patients. In a previous study, the age of patients ranged from 20 to 85 . Conversely, in our study, the age of patients ranged from one to 15 , and the average age was 4.10 years old. Almost all patients in our study had not shown secondary sexual characteristics. This explains why the expansion rate was not influenced by sex in our study.

To the best of our knowledge, this is the first research investigating the expansion rate. Although there were several studies that reported on the effectiveness of serial expansions, there were no reports regarding the differences of the expansion rate [12]. However, our study has some limitations that must be overcome with subsequent studies. First, the study was retrospective in nature. Second, no histological data existed; hence, it was not possible to prove or fully verify our hypothesis. In a further prospective study, a histological analysis should be considered as a tool to analyze the alignment of dermal contents, such as collagen and elastic fibers in the expanded flaps. Third, there was no standardization of important variables, such as endpoint of inflation. The mean endpoint of inflation was 2.35 times larger than the inserted expander, but the values varied from 0.9 to 5.25. In following studies, we recommend fixing the endpoint of inflation so that the rate of expansion can be compared more objectively. Fourth, our patient cohort was pediatrics; thus, with 
time, they would grow up. Every subsequent expansion was performed at least 15 months later than the preceding expansion. This, inevitably, presents a case where the characteristics of patients may have not been the same. However, all the serial surgeries were performed within a period of two years; hence, the results are likely not influenced too heavily from the growth of pediatric patients.

According to our data, we can perform tissue expansion more decisively when patients had undergone a previous expansion surgery at the same flap site. This would allow for an easier and shorter inflation. Additionally, if the insertion site is the trunk, we can expect the expansion to be even easier and faster.

In conclusion, we have shown that the rate of subsequent expansion is positively correlated with the number of expansions and negatively correlated with the expander size. Moreover, the expander location has also been shown to be associated with the expansion rate; faster expansion rate at the trunk. Lastly, we showed that sex did not have any association with the expansion rate.

\section{CONFLICT OF INTEREST}

No potential conflict of interest relevant to this article was reported.

\section{PATIENT CONSENT}

The patient provided written informed consent for the publication and the use of their images.

\section{REFERENCES}

1. Kang HG, Park MC, Park DH. A new modality for treating congenital melanocytic nevus: "cogwheel pattern" serial excision method. Arch Plast Surg 2014;41:418-20.
2. Rasmussen BS, Henriksen TF, Kolle SF, et al. Giant congenital melanocytic nevus: report from 30 years of experience in a single department. Ann Plast Surg 2015;74:223-9.

3. Ma T, Fan K, Li L, et al. Tissue expansion in the treatment of giant congenital melanocytic nevi of the upper extremity. Medicine (Baltimore) 2017;96:e6358.

4. Arneja JS, Gosain AK. Giant congenital melanocytic nevi. Plast Reconstr Surg 2007;120:26e-40e.

5. Tepole AB, Ploch CJ, Wong J, et al. Growing skin: a computational model for skin expansion in reconstructive surgery. J Mech Phys Solids 2011;59:2177-90.

6. Johnson PE, Kernahan DA, Bauer BS. Dermal and epidermal response to soft-tissue expansion in the pig. Plast Reconstr Surg 1988;81:390-7.

7. Jeong C, Chung HY, Lim HJ, et al. Applicability and safety of in vitro skin expansion using a skin bioreactor: a clinical trial. Arch Plast Surg 2014;41:661-7.

8. Johnson TM, Lowe L, Brown MD, et al. Histology and physiology of tissue expansion. J Dermatol Surg Oncol 1993;19:1074-8.

9. Zollner AM, Buganza Tepole A, Gosain AK, et al. Growing skin: tissue expansion in pediatric forehead reconstruction. Biomech Model Mechanobiol 2012;11:855-67.

10. Cua AB, Wilhelm KP, Maibach HI. Elastic properties of human skin: relation to age, sex, and anatomical region. Arch Dermatol Res 1990;282:283-8.

11. Nedelec B, Forget NJ, Hurtubise T, et al. Skin characteristics: normative data for elasticity, erythema, melanin, and thickness at 16 different anatomical locations. Skin Res Technol 2016;22:263-75.

12. Hudson DA, Lazarus D, Silfen R. The use of serial tissue expansion in pediatric plastic surgery. Ann Plast Surg 2000;45: 589-93. 